\title{
Clinical characteristics and outcomes for HIV-associated Diffuse Large B-cell Lymphoma in China: A retrospective single-center study
}

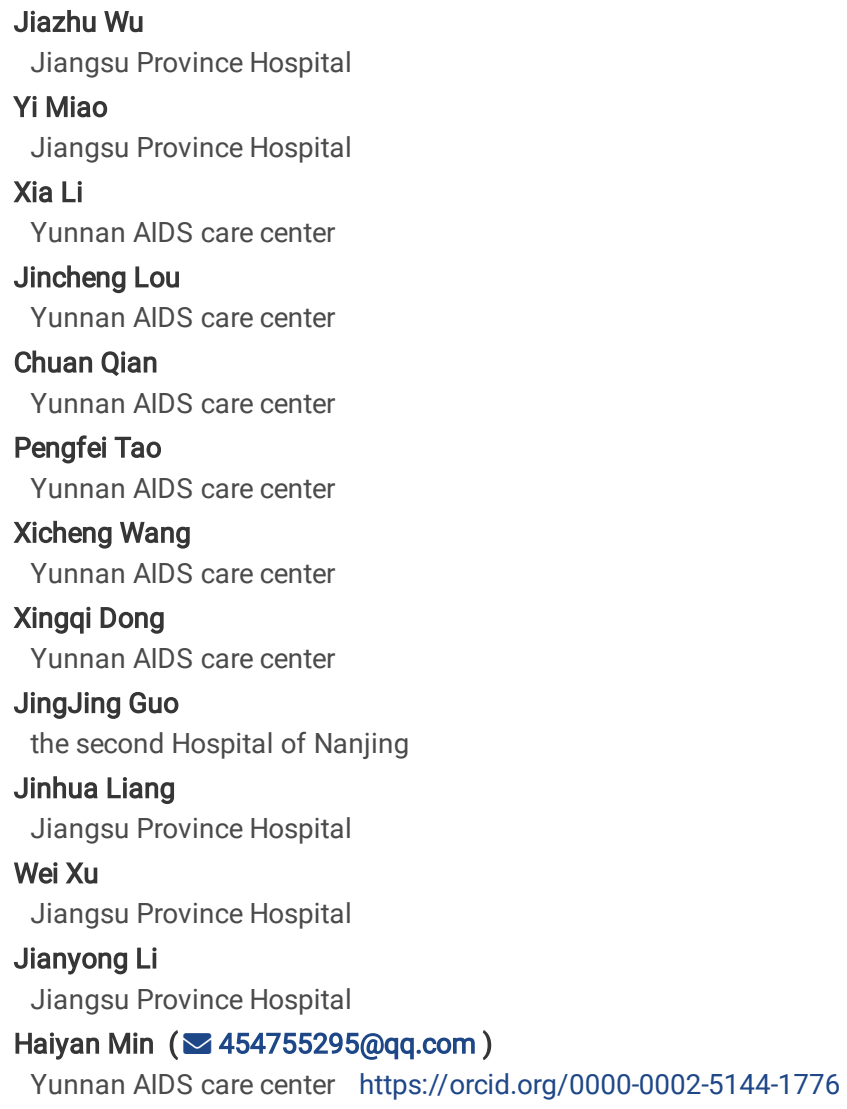




\section{Abstract}

Background: Human immunodeficiency virus (HIV) infection is associated with an increased risk of aggressive lymphoma, especially diffuse B cell lymphoma (DLBCL). We aimed to analyze characteristics and outcomes of DLBCL in HIV-associated patients. Methods: We retrospectively studied HIV-infected patients with DLBCL since 2011. Data on HIV infection and lymphoma characteristics, treatment and outcome were retrieved and analyzed. Results: In 53 patients with HIV-associated DLBCL, most patients had frequent bad performance status (PS) (74\%), elevated LDH level (94\%), B symptoms (69\%), advanced Ann Arbor (75\%), bulky tumor (72\%) and extra-nodal involvement (68\%) at diagnosis. The median CD4 T cell count was $175 / \mu$, and 17 patients were already on cART treatment. Plasma EBV DNA was detectable in 18 patients $(53 \%, 18 / 34)$. Of the patients evaluated at the end of treatment, 21 (64\%) achieved CR, 1 (3\%) achieved PR and 6 (18\%) experienced progressive disease. The 2-year progression free survival (PFS) was $50 \%$ and overall survival (OS) rate was $60 \%$. Factors associated with poor PFS in univariate analysis were unfavorable PS, high IPI score and detectable plasma EBV load. High IPI and detectable EBV load correlated with worse OS. However, only detectable plasma EBV load was the independent factor of poor prognosis for PFS (HR: 14.46, 95\% Cl [2.57-81.50]) and OS (HR: 7.47, 95\% Cl [1.48-37.59]) in a multivariate Cox regression model. Conclusions: In our population, HIV-associated DLBCL still presented aggressive characteristics and poor survival outcomes. Plasma EBV DNA could be used as a prognostic factor in HIV-infected DLBCL.

\section{Introduction}

Human immunodeficiency virus (HIV) infection is associated with an increased incidence of malignancy. HIV-associated diffuse large B cell lymphoma (DLBCL) and Burkitt's lymphoma (BL) are among the commonest cancers in HIV-positive patients [1-3]. Other non-Hodgkin's lymphomas (NHLs), including primary effusion lymphoma (PEL), plasmablastic lymphoma, KSHV-associated multicentric Castleman's disease, primary central nervous system (CNS) lymphoma and classic Hodgkin's lymphoma (cHL), are diagnosed in patients with HIV as well [1]. It has been previously studied that HIV strongly contributed to the NHL mortality, particular in acquired immunodeficiency syndrome (AIDS)-defining subtypes [4]. With the widespread administration of combination antiretroviral therapy (cART), the incidence of HIV-associated lymphoma has decreased and an improvement in the survival outcome has been observed [4, 5$]$.

DLBCL is the most common subtype of lymphoid malignancy in adults. It represents approximately $30 \%$ of $\mathrm{NHL}$ cases without $\mathrm{HIV}$ infection and $45 \%$ of cases with HIV-related lymphoma [6-8]. In a Spanish study, compared with HIV-uninfected DLBCL patients, HIV-infected patients had more aggressive features with a poorer performance status, more frequent B symptoms and more advanced Ann Arbor stages [5]. In the above study, when treated with the standard-of-care regimen rituximab plus cyclophosphamide, hydroxydaunorubicin, vincristine and prednisolone (R-CHOP), HIV-infected patients had a similar disease-free survival but a significantly worse overall survival (OS), compared to those without HIV infection [5]. In contrast, a French study revealed that HIV-infected patients with DLBCL showed survival outcomes similar to those of HIV-negative DLBCL patients [9].

Infections with Epstein-Barr virus (EBV), Hepatitis B virus (HBV), and/or Hepatitis C virus (HCV) have been reported previously in DLBCL, and the former two factors independently predicted poor prognosis [10-13]. In HIV-infected patients with DLBCL, it has been shown that elevated EBV load predicted inferior survival outcome; however, few data on HIV and HBV/HCV co-infected situation in patients with DLBCL were published [13].

The HIV epidemic appears to have obvious regional distribution in China, occurring frequently in Sichuan province and Yunnan province [14, 15]. So far, there are only few studies described the clinical features of Chinese AIDS patients with DLBCL $[16,17]$. Herein, we retrospectively analyzed the characteristics and outcomes of Chinese HIV-infected DLBCL patients in Yunnan Provincial Infectious Diseases Hospital/Yunnan AIDS Care Center.

\section{Methods}

\section{Patients}

We retrospectively included 53 HIV-infected individuals with newly diagnosed DLBCL between 2011 and 2018 in Yunnan Provincial Infectious Diseases Hospital/Yunnan AIDS Care Center, which is the largest HIV/AIDS referral hospital in southwestern China. This study was approved by institutional review board and conducted according to the declaration of Helsinki. The diagnosis was established in accordance with 2008 WHO classification. DLBCL was classified by the Hans classification algorithm. The clinical data were collected from medical records. Data of patient's demographic characteristics (gender and age), HIV-related characteristics (HIV transmission route, years of HIV infection at DLBCL diagnosis, CD4 cell count at DLBCL diagnosis and date of cART initiation), lymphoma-related characteristics (cell-of-origin subtype, Eastern Cooperative Oncology Group (ECOG) performance status score, serum lactate dehydrogenase (LDH), B symptoms, extra-nodal sites, Ann Arbor stage, international prognostic index (IPI) score, bulky tumor, bone marrow involvement and CNS-IPI score) and other related characteristics (EBV load, HBV load, HCV load, comorbidities, time from first complain to diagnosis) were available.

In this study, CART regimen included two nucleoside reverse transcriptase inhibitors and one non-nucleoside reverse transcriptase inhibitor. Patients after diagnosis of DLBCL initiated or were switched to the CART treatment with tenofovir, lamivudine, plus efavirenz or raltegravir potassium. Patients with a CD4 ${ }^{+}$ cell count below 200 cells/ $\mu$ received trimethoprim/sulphamethoxazole against Pneumocystis jiroveci as prophylactic agent. HIV-1 RNA load was monitored during chemotherapy. For patients with positive hepatitis B surface Ag (HBsAg), prophylaxis was not used because both tenofovir and lamivudine have antiviral activity against HBV. HBV DNA (cutoff $\geq 5 \times 10^{2}$ copies/ml) and HCV RNA loads (cutoff $\geq 5 \times 10^{2} \mathrm{IU} / \mathrm{ml}$ ) were assessed during chemotherapy as well. Laboratory monitoring of plasma EBV DNA (cutoff $\geq 5 \times 10^{3}$ copies $/ \mathrm{ml}$ ) load was performed in some patients.

\section{Response assessment}

Interim evaluation was performed after completion of three or four cycles of chemotherapy. After one month of the completion of all the treatment, the efficacy evaluation was conducted. Computed tomography (CT) or ${ }^{18} \mathrm{~F}$-fluorodexyglucose positron emission tomography (PET) was performed for radiological 
evaluation. Brain Magnetic Resonance Imaging (MRI) was used for CNS involvement evaluation. The 2007 revised Cheson criteria were performed to define complete response (CR), partial response (PR), progressive disease (PD) and relapse.

\section{Statistical analysis}

All statistical data were analyzed using SPSS software, version 18 or GraphPad Prism 6. Continuous variables were presented as the median with the first quartile and third quartile, and categorical variables as numbers and percentages. PFS was defined as time from DLBCL diagnosis to progression, relapse or death from any cause. OS was defined as time from DLBCL diagnosis to last follow-up or death from any cause. Survival curves were plotted using the Kaplan-Meier method and the log-rank test was used for comparison. Cox proportional regression models were performed for univariate and multivariate analyses of outcomes. $\mathrm{P}$ value $\mathbb{\otimes} 0.05$ was considered as statistically significant.

\section{Results \\ Patient characteristics}

The baseline clinical features of HIV-infected patients were summarized in Table 1. Of these patients, median age was 42 and 46 ( $87 \%$ ) of them were male. The main HIV transmission group was heterosexuals. Among 53 patients, 20 patients had a HIV infection history more than 1 year at the time of DLBCL diagnosis, and in 24 patients, DLBCL and HIV infection were diagnosed concomitantly. The median CD 4 T cell count at DLBCL diagnosis was $175 / \mu l$ (range 26-551) in these 53 patients, of whom 4 patients had a CD4 T cell count less than 50/ $\mu$ l. At DLBCL diagnosis, 17 patients were already on cART treatment. The median time from patients' first complain to diagnosis was 2 months. Most patients (74\%) had a bad performance status (PS) (ECOG PS $2-4$ ), and elevated LDH at diagnosis were present in $94 \%$. Five patients presented with 1 or 2 comorbidities (9\%). Six-nine percent of patients had B symptoms, $75 \%$ had an advanced Ann Arbor stage ( $(\mathbb{Z}-\mathbb{Z})$ and $72 \%$ had bulky tumor at diagnosis. Thirty-six patients $(68 \%)$ had extra-nodal involvement, of whom thirteen patients with more than 2 extra-nodal sites. The most frequent extra-nodal sites were the gut $(n=19)$, bone marrow $(n=7)$, stomach $(n=6)$, bone $(n=6)$, liver $(n=4)$ and/or kidney $(n=4)$. One-third of the patients had a higher central nervous system-IPI (CNS-IPI) at diagnosis. Among 34 cases with information on EBV status, EBV load was elevated $\left(5 \times 10^{3}\right.$ copies $\left./ \mathrm{ml}\right)$ in $18(53 \%)$. Of all patients, six patients $(11 \%)$ presented with positive HBsAg and four $(7.5 \%)$ presented with positive anti-HCV antibody.

\section{DLBCL treatment}

Forty-two (42/53) patients received $\mathrm{CHOP} \pm \mathrm{R}$ regimen $\rrbracket a m o n g$ which twenty-one patients received six to eight cycles $\mathrm{CHOP} \pm \mathrm{R}$ regimen. Nine of these 42 patients received $\mathrm{R}-\mathrm{CHOP}$ and seven received $\mathrm{CHOP}$ regimen, while five received $\mathrm{CHOP}$ followed by $\mathrm{R}-\mathrm{CHOP}$ or vice versa depending on their financial situation at that time. A total of twelve patients stopped $\mathrm{CHOP} \pm \mathrm{R}$ after a median cycle of 3 when symptoms improved without respect to our advices. One patient who received 4 cycles R-CMOP (cyclophosphamide, mitoxantrone, vincristine and prednisone) was switched to R-GOD (gemcitabine, oxaliplatin and dexamethasone) plus oral lenalidomide due to PD. Six patients who received less than 6 cycles $\mathrm{CHOP} \pm \mathrm{R}$ regimen died on therapy. Nine patients with high aaIPI or high-grade disease received R-EPOCH/R-DA-EPOCH (etoposide, prednisone, vincristine, cyclophosphamide and doxorubicin) regimen. Two patients received first-line R-CHOP progressed in interval evaluation and one was changed to GemOx (gemcitabine and oxaliplatin) regimen and the other was switched to GDP (gemcitabine, dexamethasone and carboplatin) regimen. One patient received four cycles R-CHOP was in PR in interval evaluation; however it was switched to R-GDP regimen due to a high SUVmax of interval PET/CT evaluation. Thirty-eight out of 53 patients received intrathecal methotrexate and cytarabine for central nervous system prophylaxis. Radiotherapy with 40-50 Gy was given in ten patients with bulky tumor】among which 4 patients received radiotherapy as consolidation after chemotherapy with $\mathrm{CR}$ and 6 patients were treated with concurrent chemoradiotherapy. Three patients were given autologous hematopoietic stem cell transplant (auto-SCT) following chemotherapy.

\section{Treatment response and outcomes}

Among forty-seven HIV-infected DLBCL patients who are evaluable in interim analysis, the overall response rate (ORR) was $88 \%$, including 22 (47\%) CR and 19 (41\%) PRs. Three patients experienced PD and three patients died before the interval evaluation. One died from hemorrhage, one from severe pleural effusion due to mycobacteriumtuberculosis and one from rapid progression of disease. Of the 33 patients evaluated at the end of treatment, 21 (64\%) achieved CR, 1 (3\%) achieved PR and 6 (18\%) experienced progressive disease (Table 2). The other 5 patients died during chemotherapy, before response could be evaluated, from sepsis $(n=4)$, or heart failure $(n=1)$. No one included in our cohort had baseline CNS involvement. With over $70 \%$ patients received CNS prophylaxis, one patient had a CNS relapse 4 months after reaching $C R$, died quickly.

The median PFS for HIV-infected DLBCL patients in our study was 24 months and median OS was not reached. The overall 2-year PFS and OS rates were 50\% $(95 \% \mathrm{Cl}[33 \%-65 \%])$ and $60 \%(95 \% \mathrm{Cl}[42 \%-74 \%])$, respectively (Fig. 1A and 1B). ECOG performance status score at least 2 (HR: 4.73, 95\% Cl [1.10-20.26]), IPI $\geq 3$ (HR: $3.65,95 \% \mathrm{Cl}$ [1.08-12.34]) and elevated plasma EBV load ( $\geq 5 \times 103$ copies/ml) (HR: 6.73, 95\% Cl [1.78-25.45]) were predictive of worse PFS, while IPI $\geq 3$ (HR: $9.12,95 \% \mathrm{Cl}[1.21-68.83]$ ) and elevated plasma EBV load ( $\geq 5 \times 103$ copies/ml) (HR: $6.26,95 \% \mathrm{Cl}$ [1.27-30.80]) were associated with worse 0S; however, in a multivariate Cox regression model, only elevated plasma EBV load was the independent factor of poorer prognosis for PFS (HR: 14.46 , $95 \% \mathrm{Cl}$ [2.57-81.50]) and OS (HR: 7.47, 95\% CI [1.48-37.59]) (Table 3). No HIV related characteristics were associated with PFS and OS. Neither HBV nor HCV coinfection was associated with prognosis. The survival curve of plasma EBV load positive patients $(n=18)$ and plasma EBV load negative patients $(n=16)$ for PFS and OS were shown in Figure 1C and 1D, reaching significantly statistical difference $(p=0.001$ and $p=0.006)$. 


\section{Discussion}

Yunnan is located in southwest China, border with Myanmar, Laos and Vietnam, and has been regarded as the most major site of HIV infection epidemic for a long time $[14,15]$. Over the decades, people living with HIV/AIDS is increasing in Yunnan, and it is still one of the provinces with highest number of HIVinfected patients in China [18].

DLBCL is the most common pathological subtype of NHL in both the general population and people living with HIV/AIDS [1-3, 19]. A large database study shows that HIV infection continues to be an independent risk factor for death among patients with lymphoma [20]. We analyzed the clinical features and survival outcomes of HIV-infected DLBCL in the setting of cART in our series. Consistent with other recent studies, HIV-infected patients with DLBCL presented with a poor performance status, a high frequency of B symptoms, elevated LDH, advanced Ann Arbor stage and high aalPI score [5, 6, 9, 17, 20]. Moreover, bulky disease was frequent. EBV infection is found in approximately $10 \%$ of HIV negative DLBCL [21]; however, in our study, $53 \%$ of patients with HIV positive DLBCL had elevated plasma EBV DNA load, suggesting a higher rate of EBV co-infection. In contrast, the frequency of HBV infection of HIV-infected patients with DLBCL was similar with their HIV-uninfected counterparts reported in Chinese population [11]. Likewise, the frequency of HCV infection was close to that of HIV negative DLBCL in Caucasian population $₫$ but lower than that in Asian population [22, 23]. Unlike previous study, in which HIV had been diagnosed a median of 15 years previously and nearly $80 \%$ had received CART at DLBCL diagnosis [9], patients in our study revealed the late HIV detection and late cART exposure. It could partially be attributed to the less awareness of HIV testing and less initiative for medical care assistance in high risk HIV population.

Before the introduction of anti-CD20 antibody rituximab, HIV-infected patients with DLBCL treated with CHOP therapy showed a similar response rate and survival as those of HIV-uninfected counterparts [24, 25]. Several retrospective studies of DLBCL patients with HIV infection treated with R-CHOP have been reported. In one study from Spain, HIV-infected DLBCL patients had a CR rate of $69 \%$ and a 5 -year OS rate of $56 \%$, similar to those of $81 \%$ and $74 \%$ of HIVuninfected patients [5]. Similarly, in a report from France, the survival outcomes of HIV-infected DLBCL patients (2-year PFS \& OS $₫ 81 \%$ and $81 \%)$ did not differ from those of HIV-uninfected patients (2-year PFS \& OS $₫ 71 \%$ and 83\%) [9]. Moreover, Sparana et al, concluded R-EPOCH was an effective regimen in HIVassociated NHL, achieving a CR rate of 73\% [26]. In this study, a total of 53 HIV-infected DLBCL patients treated between 2011 and 2018 were analyzed. Most of the patients received $\mathrm{CHOP}+\mathrm{R}$ regimen, and several patients were given $\mathrm{EPOCH}+\mathrm{R}$, based on their disease status and financial situation. Additionally, a cooperative group prospective trial reported the safety and efficacy of auto-SCT in HIV positive lymphoma including DLBCL [27]. In our study, three patients underwent auto-SCT after CR were lymphoma free. At the end of treatment, $64 \%$ of evaluable patients achieved CR. The 2 -year PFS was $50 \%$ and OS rate was $60 \%$. The response rate and survival outcome appear to be lower than others, only similar with a two-year OS rate of $63 \%$ from a German cohort, in which mostly patients treated with $\mathrm{CHOP}$-like regimen [7]. One of the reasons might be the less use of rituximab. Medical insurance has not covered the cost of rituximab until the recent years. Besides, Yunnan is a remote province and relatively economically backward, thus, some patients could not afford too much in their treatment and a few had low compliance, did not adherent to the recommended courses of treatment. As a result, the high rate of loss to follow-up (16.9\%) made our results less solid.

CNS involvement has been recognized to be more common in AIDS-related lymphomas [28]. A retrospective review of database from clinical trials presented that CNS involvement at baseline was not associated with shortened overall survival, but CNS relapse was associated with a short median OS of 1.6 months [28]. Similarly, one patient in our study had a CNS relapse and died quickly. Although the introduction of prognostic model which is used for assessing the risk of CNS disease in DLBCL has guided the CNS prophylaxis in general DLBCL population, the value of this prognostic model in HIV-infected patients of DLBCL remains unclear. Survival difference was not observed with this prognostic model in our data as most patients received intrathecal prophylaxis, suggesting the role of routine CNS prophylaxis in overcoming the unfavorable prognosis of high CNS-IPI.

It has been shown that HIV-related factors, such as a low CD4 cell count and prior history of AIDS are no longer predictive of worse survival outcome in the CART era $[29,30]$, which is consistent with our results. On the other hand, the levels of HIV-1 RNA load were negative in all the subjects during the chemotherapy. We found that unfavorable performance status, high IPI score and elevated EBV DNA load were associated with poor outcomes. Nevertheless, in multivariable analysis, only elevated plasma EBV DNA load was associated with poor outcomes.

It has been widely accepted that EBV plays an important role in the pathogenesis of lymphomas, such as BL, cHL, DLBCL and natural killer (NK)/T-cell lymphoma [31]. In the setting of HIV infection, EBV infection are more frequent, positive in $30-60 \%$ of cases compared with about $10 \%$ in the general DLBCL patients $[6,13,32,33]$. We found that of $53 \%$ of our DLBCL cases were positive for plasma EBV DNA, consistent with previously reported prevalence of EBVload in HIV associated DLBCL [13]. Its high frequency makes EBV a possible factor which contributes to the development of HIV associated lymphomas and be responsible for driving a more aggressive behavior [32]. In contrast to the fact that blood EBV DNA is not predictive of outcomes in HIV-associated HL [34], previous studies have demonstrated that tumor EBV infection status is an independent adverse predictive factor of survival among patients with HIV-infected DLBCL $[35,36]$. Meanwhile, Muncunill et al. observed that plasma EBV-load had a negative prognostic impact, and could be used as an early predictor of HIVrelated lymphoma [13]. In this study we showed plasma EBV positivity was an independent negative predictor for both PFS and OS. To our knowledge, this is one of the few studies reported with the prognostic value of plasma EBV DNA in HIV-infected DLBCL, suggesting a possibility of measuring the level of plasma EBV DNA for risk stratification.

In HIV negative DLBCL, HBsAg-positive patients have worse clinical features and poor outcomes and the presence of HCV revealed inferior OS when companied with impaired liver function [11,37]; whereas our results indicated HIV and HBV/HCV co-infected situation in DLBCL did not predict outcomes. Further studies with more samples needed to re-evaluation.

This study provides important real-world data on outcomes of HIV-infected DLBCL patients in China. A limitation of this study is the small sample size, although this is the largest series reported in China. The survival outcomes remain poor and more therapeutic approaches are warranted. Of note, EBV DNA loads at diagnosis have prognostic value and the use of EBV DNA loads in monitoring disease in patients after treatment needs to be further evaluated. 


\section{List Of Abbreviations}

HIV: Human immunodeficiency virus; DLBCL: Diffuse large B cell lymphoma; BL: Burkitt's lymphoma; NHLs: Non-Hodgkin's lymphomas;PEL: Primary effusion lymphoma; CNS: Central nervous system; CHL: Classic Hodgkin's lymphoma; AIDS: Acquired immunodeficiency syndrome; cART: Combination antiretroviral therapy; OS: Overall survival; EBV: Epstein-Barr virus; HBV: Hepatitis B virus; HCV: Hepatitis C virus; ECOG: Eastern Cooperative Oncology Group; LDH: Serum lactate dehydrogenase; IPI: International prognostic index; CT: Computed tomography; PET: ${ }^{18} \mathrm{~F}$-fluorodexyglucose positron emission tomography; MRI: Magnetic Resonance Imaging; CR: Complete response; PR: Partial response; PD: Progressive disease; PS: Performance status; auto-SCT: Autologous hematopoietic stem cell transplant; ORR: Overall response rate; NK: natural killer; GC: germinal center.

\section{Declarations}

Acknowledgements: Not applicable.

Author's contributions:JZW analyzed the data and drafted the manuscript. YM contributed to analysis and interpretation of data and revised the manuscript. JZW, YM and HYM contributed to the conception of the study. XL, JCL, CQ, PFT, XCW, XQD, JJG and JHL participated in clinical data collection. YM contributed to the data analysis. WX, JYL and HYM reviewed the manuscript and provided suggestions. All authors read and approved the final manuscript.

Availability of data and materials: The datasets analyzed during the study are available from the corresponding author on reasonable request.

Competing interests: The authors declare that they have no conflicts interest.

Consent for publication: Not applicable.

Ethics approval and consent to participate: This study was approved by Yunnan Provincial Infectious Diseases Hospital/Yunnan AIDS Care Center institutional review board and conducted according to the declaration of Helsinki. Informed consent was obtained from all participants.

Funding: This study was supported by National Natural Science Foundation of China (81370657, 81470328, 81600130, 81770166, 81720108002), Project of National Key Clinical Specialty, National Science \& Technology Pillar Program (2014BAI09B12), Jiangsu Provincial Special Program of Medical Science (BL2014086 and BE2017751), National Science and Technology Major Project (2018ZX09734007), Science Foundation for Youths of Jiangsu Province (BK20171079), and National Science \& Technology Key Program (2017ZX10202101-001-009).

\section{References}

1.Yarchoan R, Uldrick TS: HIV-Associated Cancers and Related Diseases. The New England journal of medicine 2018, 378(22):2145.

2.Meister A, Hentrich M, Wyen C, Hubel K: Malignant lymphoma in the HIV-positive patient. European journal of haematology 2018, 101(1):119-126.

3.Gopal S, Patel MR, Yanik EL, Cole SR, Achenbach CJ, Napravnik S, Burkholder GA, Reid EG, Rodriguez B, Deeks SG et al: Temporal trends in presentation and survival for HIV-associated lymphoma in the antiretroviral therapy era. Journal of the National Cancer Institute 2013, 105(16):1221-1229.

4.Howlader N, Shiels MS, Mariotto AB, Engels EA: Contributions of HIV to Non-Hodgkin Lymphoma Mortality Trends in the United States. Cancer epidemiology, biomarkers \& prevention: a publication of the American Association for Cancer Research, cosponsored by the American Society of Preventive Oncology 2016, 25(9):1289-1296.

5.Baptista MJ, Garcia O, Morgades M, Gonzalez-Barca E, Miralles P, Lopez-Guillermo A, Abella E, Moreno M, Sancho JM, Feliu E et al: HIV-infection impact on clinical-biological features and outcome of diffuse large B-cell lymphoma treated with R-CHOP in the combination antiretroviral therapy era. Aids 2015, 29(7):811-818.

6.Li S, Young KH, Medeiros LJ: Diffuse large B-cell lymphoma. Pathology 2018, 50(1):74-87.

7.Schommers P, Hentrich M, Hoffmann C, Gillor D, Zoufaly A, Jensen B, Bogner JR, Thoden J, Wasmuth JC, Wolf T et al: Survival of AlDS-related diffuse large B-cell lymphoma, Burkitt lymphoma, and plasmablastic lymphoma in the German HIV Lymphoma Cohort. British journal of haematology 2015, 168(6):806810.

8.Miao Y, Medeiros LJ, Xu-Monette ZY, Li J, Young KH: Dysregulation of Cell Survival in Diffuse Large B Cell Lymphoma: Mechanisms and Therapeutic Targets. Frontiers in Oncology 2019, 9.

9.Besson C, Lancar R, Prevot S, Algarte-Genin M, Delobel P, Bonnet F, Meyohas MC, Partisani M, Oberic L, Gabarre J et al: Outcomes for HIV-associated diffuse large B-cell lymphoma in the modern combined antiretroviral therapy era. Aids 2017, 31(18):2493-2501.

10.Wu L, Ehlin-Henriksson B, Zhou X, Zhu H, Ernberg I, Kis LL, Klein G: Epstein-Barr virus (EBV) provides survival factors to EBV(+) diffuse large B-cell lymphoma (DLBCL) lines and modulates cytokine induced specific chemotaxis in EBV(+) DLBCL. Immunology 2017, 152(4):562-573.

11.Deng L, Song Y, Young KH, Hu S, Ding N, Song W, Li X, Shi Y, Huang H, Liu W et al: Hepatitis B virus-associated diffuse large B-cell lymphoma: unique clinical features, poor outcome, and hepatitis B surface antigen-driven origin. Oncotarget 2015, 6(28):25061-25073. 
12.Tsutsumi Y, Nakayama C, Kamada K, Kikuchi R, Kudo D, Ito S, Matsuoka S, Shiratori S, Yamamoto Y, Naruse H et al: Efficacy and prognosis of antiviral therapy on hepatitis C following treatment of lymphoma in HCV-positive diffuse large-cell lymphoma. Annals of hematology 2017, 96(12):2057-2061.

13.Muncunill J, Baptista MJ, Hernandez-Rodriguez A, Dalmau J, Garcia O, Tapia G, Moreno M, Sancho JM, Martinez-Picado J, Feliu E et al: Plasma EBV-load as an early biomarker and prognostic factor of HIV-related lymphomas. Clinical infectious diseases: an official publication of the Infectious Diseases Society of America 2018.

14.Lu L, Jia M, Ma Y, Yang L, Chen Z, Ho DD, Jiang Y, Zhang L: The changing face of HIV in China. Nature 2008, 455(7213):609-611.

15.Jia M, Luo H, Ma Y, Wang N, Smith K, Mei J, Lu R, Lu J, Fu L, Zhang Q et al: The HIV epidemic in Yunnan Province, China, 1989-2007. Journal of acquired immune deficiency syndromes 2010, 53 Suppl 1:S34-40.

16.Xiao J, Du S, Dai G, Gao G, Yang D, Zhao H: Efficacy and tolerability of chemotherapy in Chinese patients with AIDS-related Burkitt lymphoma and diffuse large B-cell lymphoma: An observational study. Scientific reports 2017, 7(1):1905.

17.Shen Y, Zhang R, Liu L, Shen Y, Song W, Qi T, Tang Y, Wang Z, Guan L, Lu H: Clinical and prognostic analysis of 78 patients with human immuno-deficiency virus associated non-Hodgkin's lymphoma in Chinese population. Infectious agents and cancer 2017, 12:7.

18.Chen M, Jia MH, Ma YL, Luo HB, Chen HC, Yang CJ, Dai J, Yang L, Dong LJ, Lu R et al: The changing HIV-1 genetic characteristics and transmitted drug resistance among recently infected population in Yunnan, China. Epidemiology and infection 2018, 146(6):775-781.

19.Ji Y, Lu H: Malignancies in HIV-Infected and AIDS Patients. Advances in experimental medicine and biology 2017, 1018:167-179.

20.Han X, Jemal A, Hulland E, Simard EP, Nastoupil L, Ward E, Flowers CR: HIV Infection and Survival of Lymphoma Patients in the Era of Highly Active Antiretroviral Therapy. Cancer epidemiology, biomarkers \& prevention: a publication of the American Association for Cancer Research, cosponsored by the American Society of Preventive Oncology 2017, 26(3):303-311.

21. Healy JA, Dave SS: The Role of EBV in the Pathogenesis of Diffuse Large B Cell Lymphoma. Current topics in microbiology and immunology 2015, 390(Pt 1):315-337.

22.de Sanjose S, Benavente Y, Vajdic CM, Engels EA, Morton LM, Bracci PM, Spinelli JJ, Zheng T, Zhang Y, Franceschi S et al: Hepatitis C and non-Hodgkin lymphoma among 4784 cases and 6269 controls from the International Lymphoma Epidemiology Consortium. Clinical gastroenterology and hepatology: the official clinical practice journal of the American Gastroenterological Association 2008, 6(4):451-458.

23.Chen YY, Huang CE, Liang FW, Lu CH, Chen PT, Lee KD, Chen CC: Prognostic impact of hepatitis C virus infection in patients with diffuse large B-cell lymphoma treated with immunochemotherapy in the context of a novel prognostic index. Cancer epidemiology 2015, 39(3):382-387.

24.Navarro JT, Lloveras N, Ribera JM, Oriol A, Mate JL, Feliu E: The prognosis of HIV-infected patients with diffuse large B-cell lymphoma treated with chemotherapy and highly active antiretroviral therapy is similar to that of HIV-negative patients receiving chemotherapy. Haematologica 2005, 90(5):704-706.

25.Weiss R, Mitrou P, Arasteh K, Schuermann D, Hentrich M, Duehrsen U, Sudeck H, Schmidt-Wolf IG, Anagnostopoulos I, Huhn D: Acquired immunodeficiency syndrome-related lymphoma: simultaneous treatment with combined cyclophosphamide, doxorubicin, vincristine, and prednisone chemotherapy and highly active antiretroviral therapy is safe and improves survival-results of the German Multicenter Trial. Cancer 2006, 106(7):1560-1568.

26.Sparano JA, Lee JY, Kaplan LD, Levine AM, Ramos JC, Ambinder RF, Wachsman W, Aboulafia D, Noy A, Henry DH et al: Rituximab plus concurrent infusional EPOCH chemotherapy is highly effective in HIV-associated B-cell non-Hodgkin lymphoma. Blood 2010, 115(15):3008-3016.

27.Alvarnas JC, Le Rademacher J, Wang Y, Little RF, Akpek G, Ayala E, Devine S, Baiocchi R, Lozanski G, Kaplan L et al: Autologous hematopoietic cell transplantation for HIV-related lymphoma: results of the BMT CTN 0803/AMC 071 trial. Blood 2016, 128(8):1050-1058.

28.Barta SK, Joshi J, Mounier N, Xue X, Wang D, Ribera JM, Navarro JT, Hoffmann C, Dunleavy K, Little RF et al: Central nervous system involvement in AIDSrelated lymphomas. British journal of haematology 2016, 173(6):857-866.

29.Miralles P, Berenguer J, Ribera JM, Rubio R, Mahillo B, Tellez MJ, Lacruz J, Valencia E, Santos J, Rodriguez-Arrondo F et al: Prognosis of AIDS-related systemic non-Hodgkin lymphoma treated with chemotherapy and highly active antiretroviral therapy depends exclusively on tumor-related factors. Journal of acquired immune deficiency syndromes 2007, 44(2):167-173.

30.Barta SK, Samuel MS, Xue X, Wang D, Lee JY, Mounier N, Ribera JM, Spina M, Tirelli U, Weiss R et al: Changes in the influence of lymphoma- and HIVspecific factors on outcomes in AIDS-related non-Hodgkin lymphoma. Annals of oncology: official journal of the European Society for Medical Oncology 2015, 26(5):958-966.

31.Vockerodt M, Yap LF, Shannon-Lowe C, Curley H, Wei W, Vrzalikova K, Murray PG: The Epstein-Barr virus and the pathogenesis of lymphoma. The Journal of pathology 2015, 235(2):312-322.

32.Linke-Serinsoz E, Fend F, Quintanilla-Martinez L: Human immunodeficiency virus (HIV) and Epstein-Barr virus (EBV) related lymphomas, pathology view point. Seminars in diagnostic pathology 2017, 34(4):352-363. 
33.Morton LM, Kim CJ, Weiss LM, Bhatia K, Cockburn M, Hawes D, Wang SS, Chang C, Altekruse SF, Engels EA et al: Molecular characteristics of diffuse large B-cell lymphoma in human immunodeficiency virus-infected and -uninfected patients in the pre-highly active antiretroviral therapy and pre-rituximab era. Leukemia \& lymphoma 2014, 55(3):551-557.

34.Ul-Haq I, Dalla Pria A, Suardi E, Pinato DJ, Froeling F, Forni J, Randell P, Bower M: Blood Epstein-Barr virus DNA does not predict outcome in advanced HIVassociated Hodgkin lymphoma. Medical oncology 2018, 35(4):53.

35.Chao C, Silverberg MJ, Martinez-Maza O, Chi M, Abrams DI, Haque R, Zha HD, McGuire M, Xu L, Said J: Epstein-Barr virus infection and expression of B-cell oncogenic markers in HIV-related diffuse large B-cell Lymphoma. Clinical cancer research: an official journal of the American Association for Cancer Research 2012, 18(17):4702-4712.

36.Chao C, Silverberg MJ, Chen LH, Xu L, Martinez-Maza O, Abrams DI, Zha HD, Haque R, Said J: Novel tumor markers provide improved prediction of survival after diagnosis of human immunodeficiency virus (HIV)-related diffuse large B-cell lymphoma. Leukemia \& lymphoma 2018, 59(2):321-329.

37.Dlouhy I, Torrente MA, Lens S, Rovira J, Magnano L, Gine E, Delgado J, Balague O, Martinez A, Campo E et al: Clinico-biological characteristics and outcome of hepatitis C virus-positive patients with diffuse large B-cell lymphoma treated with immunochemotherapy. Annals of hematology 2017, 96(3):405-410.

\section{Tables}

\section{Table 1: Clinical characteristics of HIV-infected patients with DLBCL}


$\mathrm{N}=53(\%) \quad$ Median (1 $1^{\text {st }}-3^{\text {rd }}$ quartile)

\section{Demographics}

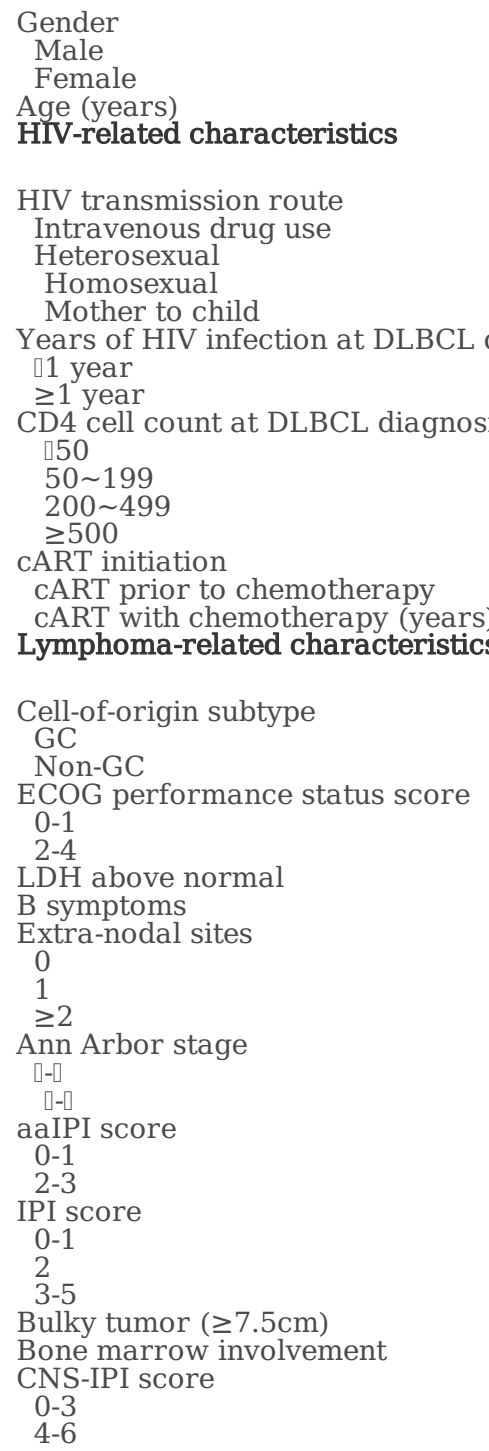

Other related characteristics

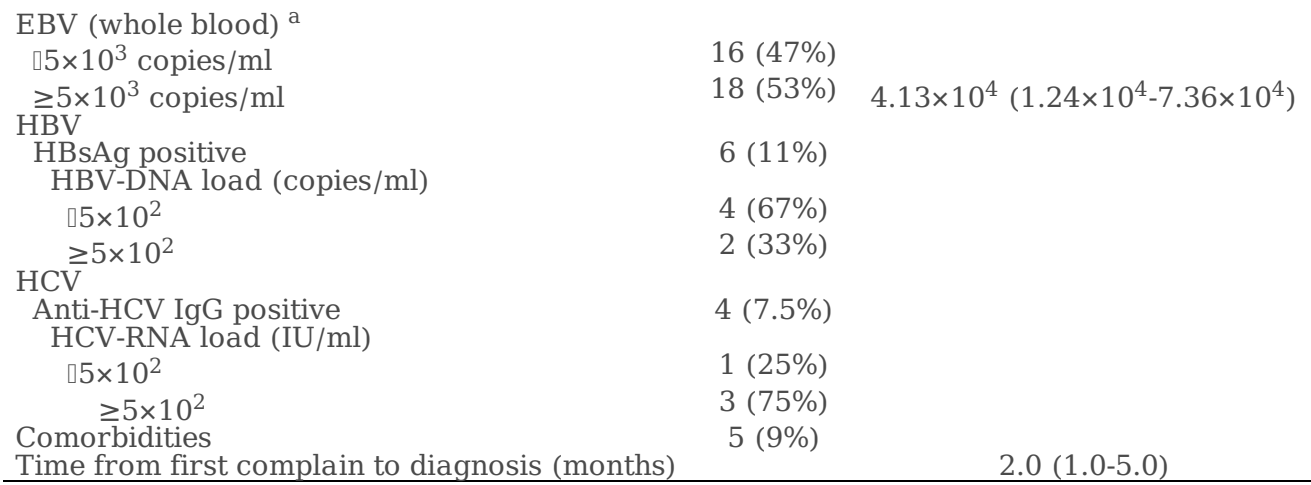

$46(87 \%)$

$7(13 \%)$

$42(35.0-51.0)$

$5(9 \%)$

$43(81 \%)$

$4(8 \%)$

$1(2 \%)$

$33(62 \%) \quad 0.08(0.04-0.24)$

$20(38 \%) \quad 5.18(2.07-9.05)$

$4(7 \%)$

$25(47 \%)$

$21(40 \%)$

$3(6 \%)$

$5(33.5-47.0)$

$132(100-160)$

$289(247.0-346.0)$

$17(32 \%)$

$36(68 \%)$

$1.64(0.48-4.64)$

$37(70 \%)$

$16(30 \%)$

$14(26 \%)$

$39(74 \%)$

$51(94 \%)$

$37(69 \%)$

$352(268.5-628.5)$

$17(32 \%)$

$23(43 \%)$

$13(25 \%)$

$13(25 \%)$

$40(75 \%)$

$9(17 \%)$

$38(72 \%)$

0

$1(2 \%)$

$5(9 \%)$

$38(72 \%)$

$7(13 \%)$

$40(75 \%)$

$13(25 \%)$
Time from first complain to diagnosis (months)

HIV: human immunodeficiency virus; DLBCL: diffuse large B-cell lymphoma; cART: combination antiretroviral therapy; GC: germinal center; ECOG: Eastern Cooperative Oncology Group; LDH: lactate dehydrogenase; B symptoms: fever, night sweats, weight loss, fatigue, and swelling in lymph nodes; CNS: central nervous system; ${ }^{\mathrm{a}}$ : numbers of missing values, ${ }^{\mathrm{a}}: 19$. 
Table 2: Evaluation following chemotherapy in HIV-infected patients with DLBCL

\begin{tabular}{lcc}
\hline & Interval evaluation ${ }^{a}$ & At the end of treatment \\
& $\mathrm{N}=53$ & $\mathrm{~N}=53$ \\
\hline Able to evaluate & $\mathrm{N}=47(\%)$ & $\mathrm{N}=33(\%)$ \\
& & \\
Complete response & $22(47 \%)$ & $21(64 \%)$ \\
Partial response & $19(41 \%)$ & $1(3 \%)$ \\
Stable disease & 0 & $6(18 \%)$ \\
Progressive disease & $3(6 \%)$ & $5(15 \%)$ \\
Death b & $3(6 \%)$ & $\mathrm{N}=17(\%)$ \\
Unable to evaluate & $\mathrm{N}=6$ & 2 \\
Ongoing treatment & 0 & 15 \\
Having stopped treatment ${ }^{\mathrm{c}}$ & 6 & \\
\hline
\end{tabular}

HIV: human immunodeficiency virus; DLBCL: diffuse large B-cell lymphoma;

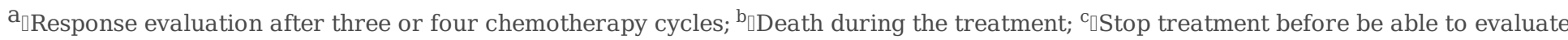
response to chemotherapy could be attributed to personal willingness, and nine cases end up in losing to follow-up.

Table 3: Cox univariable and multivariable analyses of PFS and OS in HIV-infected patients with DLBCL 


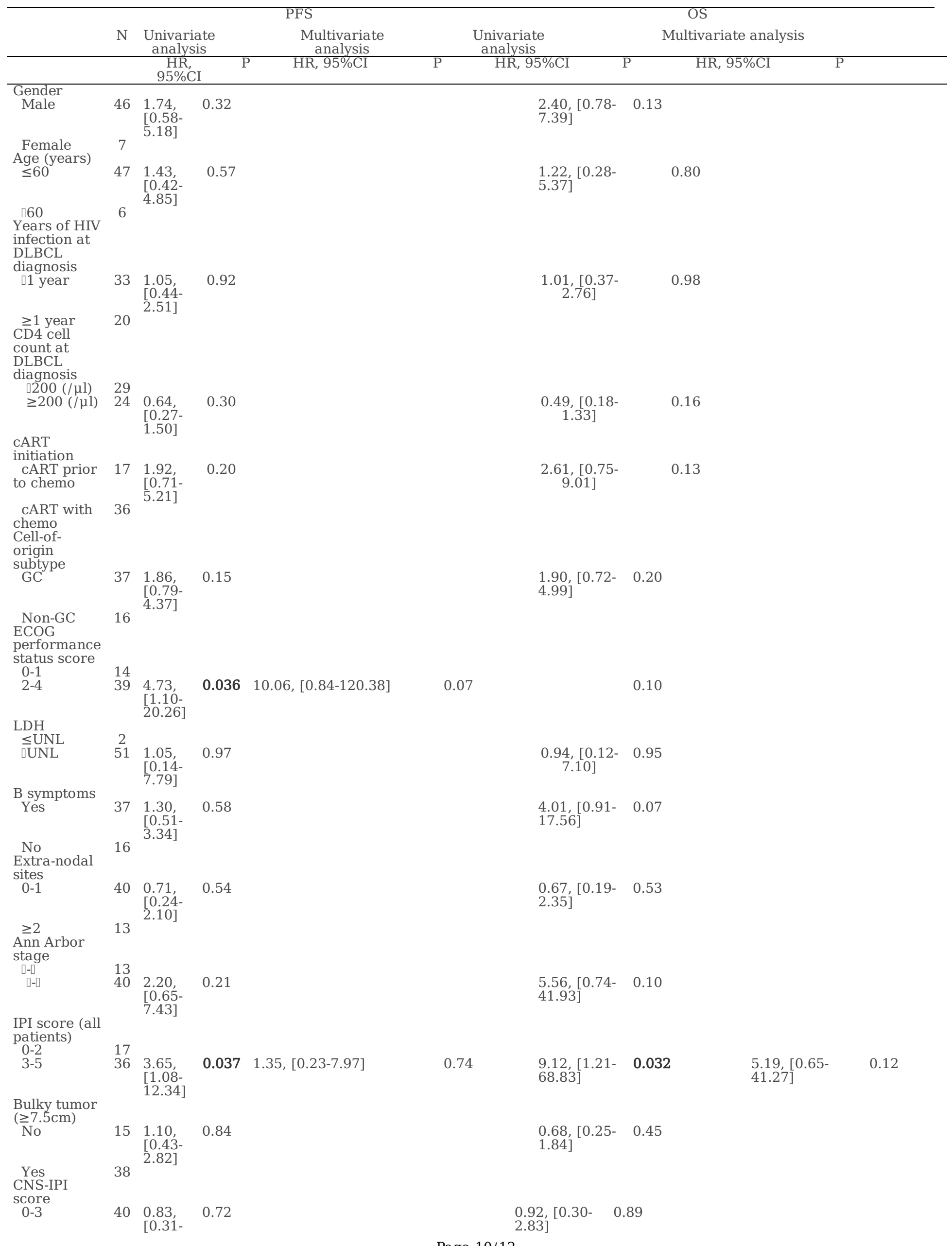




\begin{tabular}{|c|c|c|c|c|c|c|c|c|c|}
\hline $\begin{array}{c}4-6 \\
\text { EBV }\end{array}$ & 13 & $2.25]$ & & & & & & & \\
\hline $\begin{array}{l}\text { (serum) }^{a} \\
\square 5 \times 10^{3}\end{array}$ & 16 & & & & & & & & \\
\hline $\begin{array}{c}\text { coples } / \mathrm{ml} \\
\geq 5 \times 10^{3} \\
\text { copies } / \mathrm{ml}\end{array}$ & 18 & $\begin{array}{l}6.73, \\
{[1.78-} \\
25.45]\end{array}$ & 0.005 & $14.46,[2.57-81.50]$ & 0.002 & $\begin{array}{l}6.26,[1.27- \\
30.80]\end{array}$ & 0.024 & $\begin{array}{l}7.47 \\
{[1.48-} \\
37.59]\end{array}$ & 0.015 \\
\hline $\begin{array}{l}\text { HBV } \\
\text { HBsAg } \\
\text { positive }\end{array}$ & 6 & $\begin{array}{l}0.80 \\
{[0.19-} \\
3.43]\end{array}$ & 0.76 & & & $\begin{array}{l}0.48,[0.06- \\
3.65]\end{array}$ & 0.48 & & \\
\hline $\begin{array}{l}\text { HBsAg } \\
\text { negative } \\
\text { HCV }\end{array}$ & 47 & & & & & & & & \\
\hline $\begin{array}{l}\text { Anti-HCV } \\
\text { positive }\end{array}$ & 4 & $\begin{array}{l}1.53 \\
{[0.35-} \\
6.65]\end{array}$ & 0.59 & & & $\begin{array}{l}1.96,[0.44- \\
8.65]\end{array}$ & 0.38 & & \\
\hline $\begin{array}{l}\text { Anti-HCV } \\
\text { negative }\end{array}$ & 49 & & & & & & & & \\
\hline
\end{tabular}

HIV: human immunodeficiency virus; DLBCL: diffuse large B-cell lymphoma; PFS: progression free survival; OS: overall survival; HR: hazard ratio; CI: confidence interval; cART: combination antiretroviral therapy; GC: germinal center; ECOG: Eastern Cooperative Oncology Group; LDH: lactate dehydrogenase; UNL: upper normal limit; B symptoms: fever, night sweats, weight loss, fatigue, and swelling in lymph nodes; CNS: central nervous system; ${ }^{a}$ : numbers of missing values, ${ }^{a}: 19$.

\section{Figures}

PFS

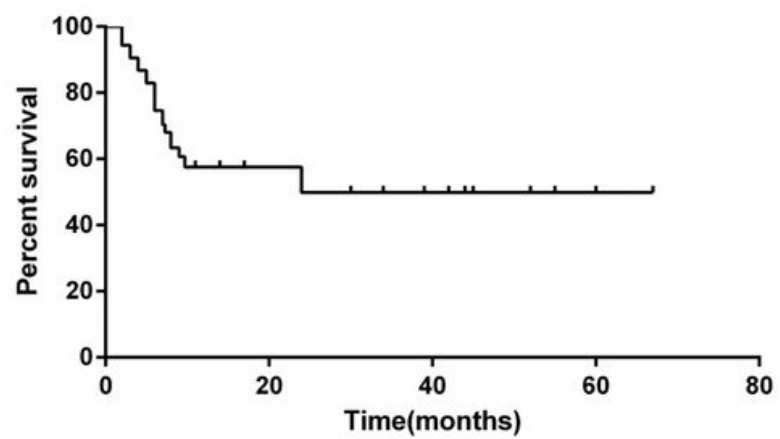

A

PFS

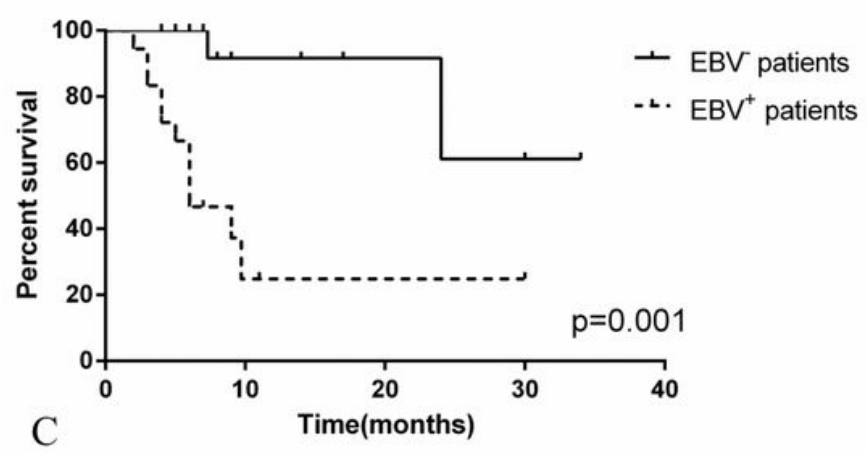

os

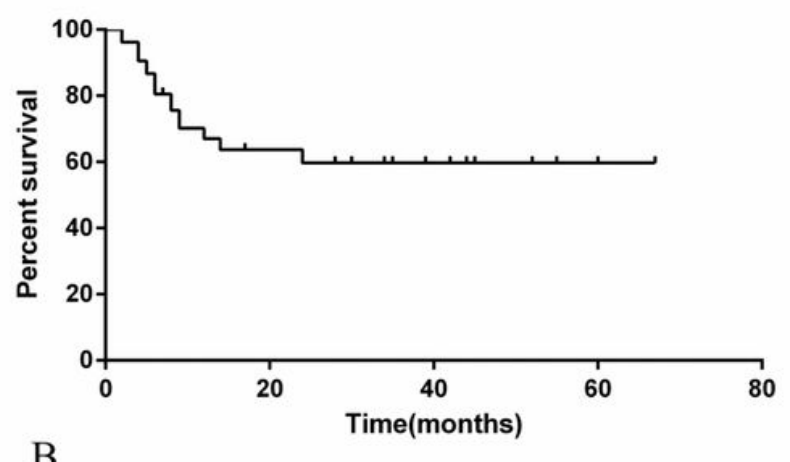

os

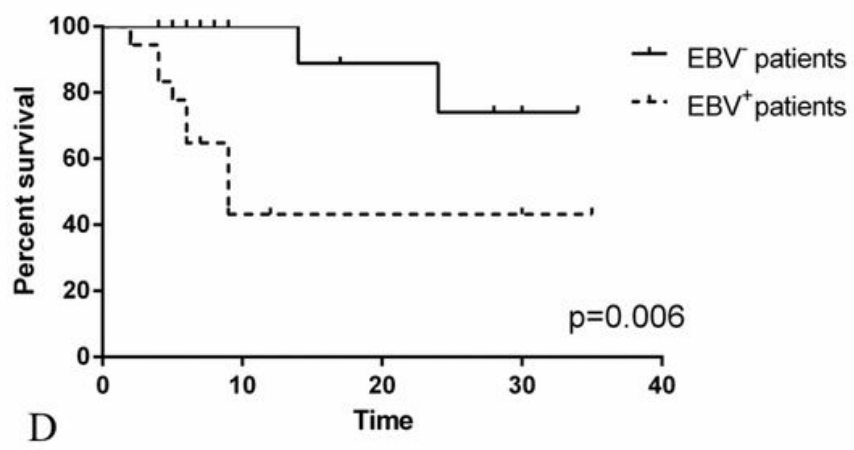

\section{Figure 1}

Figure 1: Progression-free and overall survival in HIV-infected patients with DLBCL Panel A showed the PFS for HIV-infected DLBCL patients. Panel B showed the OS for HIV-infected DLBCL patients. Panel C showed the PFS for plasma EBV load positive patients and plasma EBV load negative patients. Panel D 
showed the OS for plasma EBV load positive patients and plasma EBV load negative patients. PFS: progression free survival; OS: overall survival; DLBCL: diffuse large B cell lymphoma. 